Gabe, E. J., Le Page, Y., Charland, J.-P., Lee, F. L. \& White, P. S. (1989). J. Appl. Cryst. 22, 384-387.

Hanton, L. R., Hunter, C. A. \& Purvis, D. H. (1992). J. Chem. Soc. Chem. Commun. pp. 1134-1136.

Hardy, A. D. U. \& MacNicol, D. D. (1976). J. Chem. Soc. Perkin Trans. 2, pp. 1140-1142.

Johnson, C. K. (1976). ORTEPII. Report ORNL-5138. Oak Ridge National Laboratory, Tennessee, USA.

Larson, A. C. (1970). Crystallographic Computing, edited by F. R. Ahmed, S. R. Hall \& C. P. Huber, pp. 291-294. Copenhagen: Munksgaard.

Ovchinnikov, Yu. E., Shklover, V. E., Struchkov, Yu. T., Dement'ev, V. V., Frunze, T. M. \& Antipova, B. A. (1987). J. Organomet. Chem. $335,157-166$

Rzepa, H. S., Webb, M. L., Slawin, A. M. Z. \& Williams, D. J. (1991). J. Chem. Soc. Chem. Commun. pp. 765-768.

Spek, A. L. (1991). PLUTON. Molecular Graphics Program. Univ. of Utrecht, The Netherlands.

Toda, F., Tanaka, K., Nagamatsu, S. \& Mak, T. C. W. (1985). Isr. J. Chem. 25, 346-352.

Acta Cryst. (1994). C50, 73-77

\section{Self Inclusion in a Calix[5]arene Structure; Structure of the Cone Conformer of a Pentahydroxy-p-tert-butylcalix[5]arene}

\author{
John F. Gallagher $†$ and George Ferguson* \\ Department of Chemistry and Biochemistry, \\ University of Guelph, Guelph, Ontario, \\ Canada NIG 2WI
}

VolKer BöHmER AND Dagmar KRAFT

Institut für Organische Chemie, Becher Weg 34 SB1, Johannes Gutenberg Universtät, 6500 Mainz, Germany

(Received 22 February 1993; accepted 6 July 1993)

\begin{abstract}
The pentahydroxy-p-tert-butylcalix[5]arene, 5,11,17,23,29-penta-tert-butylhexacyclo[25.3.1.1 $1^{3,7} \cdot 1^{9,13} \cdot 1^{15,19}$.-

$1^{21,25}$ ]pentatriaconta-1(31),3,5,7(35),9,11,13(34),15,17,19(33),21,23,25(32),27,29-pentadecaene-31,32,33,34,-

35-pentaol- $n$-hexane $(1 / 0.3), \mathrm{C}_{55} \mathrm{H}_{70} \mathrm{O}_{5} .0 .3 \mathrm{C}_{6} \mathrm{H}_{14}$ (I), adopts an open distorted chalice-shaped conformation in the solid state, primarily through $\mathrm{O}-\mathrm{H} \cdots \mathrm{O}$ intramolecular hydrogen bonding involving all of the aromatic hydroxyl groups. The five phenolic $\mathrm{O} \cdots \mathrm{O}$ separations are in the range 2.735 (7)-2.880 (8) $\AA$ [mean 2.793 (8) $\AA$ ], with all hydroxyl $\mathrm{H}$ atoms disordered equally over two sites.

$\dagger$ Present address: University Chemical Laboratories, Lensfield Road, Cambridge CB2 1EW, England.
\end{abstract}

The aromatic rings are tilted back from the calixarene cavity producing a pentagonal-shaped cavity in which the tert-butyl group of a neighboring calix [5]arene related by a $c$-glide is enclathrated. This self-inclusion process extends through the lattice as a one-dimensional molecular 'zipper'.

\section{Comment}

The structural chemistry of calixarenes continues to be of immense interest primarily because of their recognized practical use as organic guest-host systems (Gutsche, 1989; Vicens \& Böhmer, 1991). This is of fundamental importance in the quest for a better understanding of natural molecular-recognition processes.

We are currently studying the molecular structures of calix[4]arenes and calix[6]arenes, and their metal complexes. Several of these have been shown to enclathrate small organic molecules within the molecular cavity, e.g. dichloromethane (Böhmer et al., 1993), ethanol (Ferguson, Gallagher \& Pappalardo, 1993) and acetonitrile (McKervey, Seward, Ferguson \& Ruhl, 1986). Until recently, there have been few structural studies reported for calix[5]arenes, mainly because of difficulties with synthesis and purification. Herein we report the structure of (I).0.3(n-hexane).

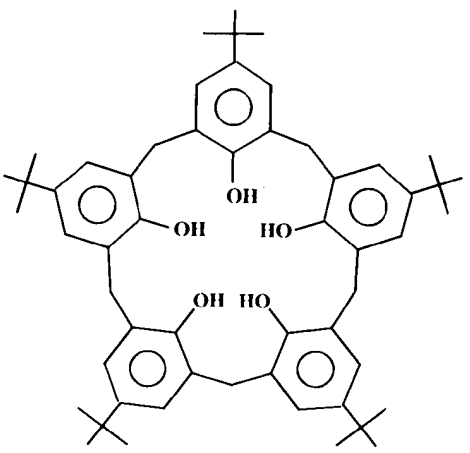

(I)

Our X-ray analysis of (I) shows that it adopts a distorted chalice conformation in the solid state (Figs. 1 and 2); the tert-butyl group on ring $C$ is disordered over two orientations. The major conformation-determining feature in this molecule is the presence of $\mathrm{O}-\mathrm{H} \cdots \mathrm{O}$ intramolecular hydrogen bonding involving all five aromatic hydroxyl groups; the $\mathrm{O}$. . O separations are in the range $2.735(7)$ 2.880 (8) $\AA$ [mean 2.793 (8) $\AA$ ]. Difference maps show that all five hydroxyl $\mathrm{H}$ atoms are disordered equally over two orientations; a similar situation was observed with the hydroxyl $\mathrm{H}$ atoms in pentahydroxycalix [5]arene acetone solvate, (II) (Coruzzi, Andreetti, Bocchi, Pochini \& Ungaro, 1982).

The conformation of (I) is defined by the angles which the aromatic rings make with the plane of the five methylene $C$ atoms which link them, viz. 149.7 (3) (A), 136.0 (3) 


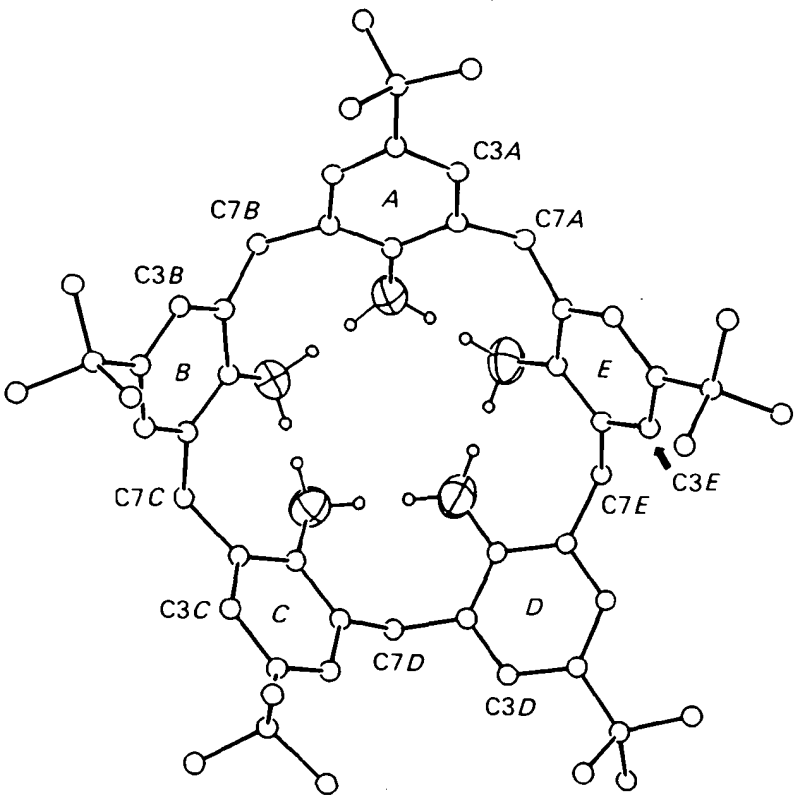

Fig. 1. An ORTEPII view of the chalice conformer of molecule (I) with our numbering scheme; the $\mathrm{O}$ atoms are shown with thermal ellipsoids drawn at the $50 \%$ probability level. For clarity, the $\mathrm{C}$ and $\mathrm{H}$ atoms are drawn as small spheres of arbitrary size.
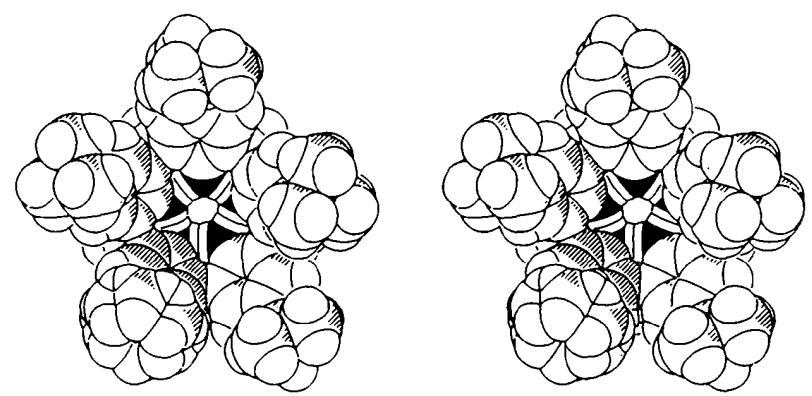

Fig. 2. A stereoview of the molecular cavity in tert-butylcalix[5]arene, with atoms drawn as their van der Waals spheres; the tert-butyl group on ring $C$ is disordered.

$(B), 118.6(3)(C), 139.8(3)(D)$ and $123.8(3)^{\circ}(E)$ (interplanar angles $>90^{\circ}$ indicate that the aromatic ring system is tilted so that its tert-butyl group is directed away from the ring cavity). Three of the aromatic rings $(A, B, D)$ are tilted so that their tert-butyl groups are pitched well away from the pentagonal-shaped calix cavity compared with those of $C$ and $E$ (Figs. 1 and 2). Ugozzoli \& Andreetti (1992) recently proposed a new approach for the assignment of the molecular conformations of calix $[n]$ arenes ( $n$ $=4-8$ ) using the values of the torsion angles involving the methylene $\mathrm{C}$ atoms, to which they assign the labels $\varphi$ and $\chi$. The $\varphi$ and $\chi$ values for these torsion angles in (I) are listed in Table 4 and are similar to those reported for pentahydroxycalix [5]arene-acetone ( $1 / 2$ solvate complex), (II) (Coruzzi et al., 1982; Ugozzoli \& Andreetti,
1992); the ranges in $\varphi$ and $\chi$ values in (II) are from 77 (1) to $96(1)^{\circ}$ and from $-81(1)$ to $-96(1)^{\circ}$, respectively.

The calix[5]arene cavity enclathrates the tert-butyl group of a neighbouring molecule related by a $c$-glide operation, giving rise to a self-inclusion complex which extends through the lattice as a one-dimensional molecular zipper, propagated along the $c$-glide planes (Fig. 3 ). Aromatic ring $A$ (to which the tert-butyl group involved in the self-inclusion process is bonded) is tilted back furthest from the plane defined by the five methylene $C$ atoms $\left[149.7(3)^{\circ}\right]$, presumably due to steric interactions and intermolecular packing effects. The shortest C...C intermolecular contacts between the enclathrated tert-butyl moiety and those of the 'cup' $\mathrm{C}$ atoms involve C9A with $\mathrm{C} 2 C[3.55(2) \AA]$ and $\mathrm{C} 6 B$ [3.57 (2) $\mathrm{A}]$. Disordered partial-occupancy $n$-hexane molecules of crystallization ( 0.3 per calix[5]arene), are present in the lattice, situated about twofold axes in what would have been voids between molecules of (I).
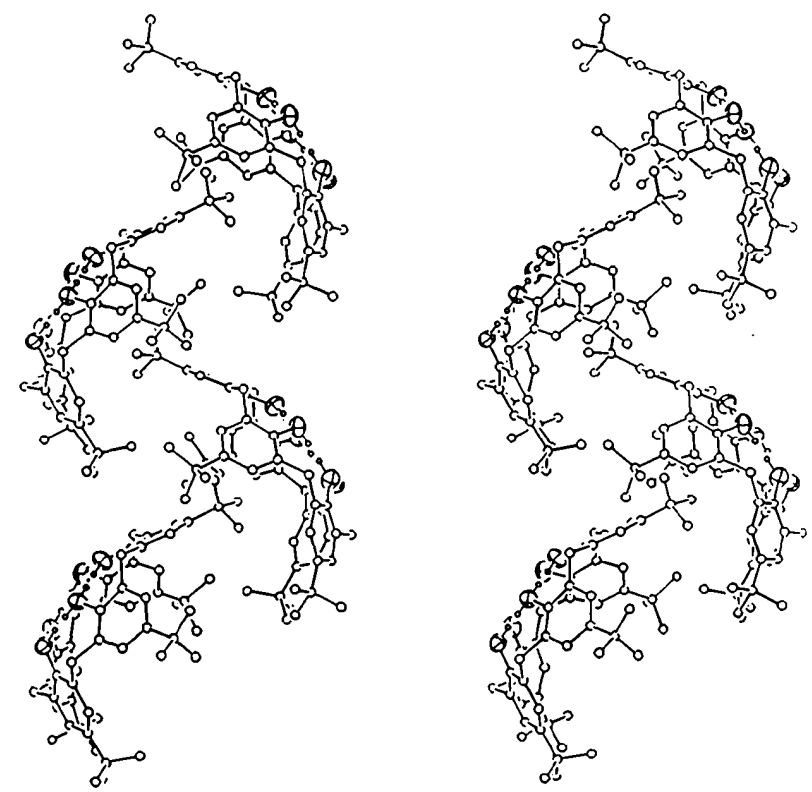

Fig. 3. An ORTEPII stereoview of a chain of molecules in (I) which results from the $c$-glide symmetry operation.

A search of the 1992 release of the Cambridge Structural Database (Allen, Kennard \& Taylor, 1983) shows that there have been previous reports on four calix[5]arenes, namely: (II) pentahydroxycalix [5]areneacetone (1/2 solvate complex) (Coruzzi et al., 1982); (III) pentahydroxy-p-tert-butylcalix[5]arene-tetralin (1/1 inclusion complex) (Juneja et al., 1992); (IV) pentahydroxy-p-1,1,3,3-tetramethylbutylcalix[5] arene and (V) pentahydroxy- $p$-1,1,3,3-tetramethylbutylcalix [5]arenetoluene (1/1 inclusion complex) (Perrin \& Lecocq, 1991). The orientation of the aromatic rings and $\mathrm{O} \cdots \mathrm{O}$ separations between proximally related $O$ atoms are summarized 
in Table 3 for the calix[5]arene structure determinations for which data are available. The $\mathrm{O} \cdots \mathrm{O}$ separations are similar in all five determinations and in the range 2.7$2.9 \AA$. The range in aromatic ring orientation angles is greatest in our present molecule, (I), presumably due to the process of self inclusion.

Perrin \& Lecocq (1991) report that two types of inclusion compounds have been found with calixarenes, with the guest either outside (as a solvate) or enclathrated within the calixarene cones [frequently described as cryptato-cavitate or tubulato-intercalato clathrates using the nomenclature proposed by Weber \& Josel (1983)]. We have reported recently a third type of inclusion in calixarenes, in a partial-cone calix[4] arene conformer where one of the aromatic rings is rotated so that its pyridine moiety is tightly accommodated inside the hydrophobic cavity as a self-inclusion monomer (Pappalardo et al., 1992). The present structure is best described as a selfinclusion polymer (Fig. 3).

We have seen this self-inclusion effect previously in the structures of (i) a diester derivative of a calix[4]arene (Ferguson, Gallagher, McKervey \& Moran, 1993) and (ii) a phthaloyl derivative of a calix[6]arene (Kraft, Böhmer, Vögt, Ferguson \& Gallagher, 1993), but (I) provides the first example of self inclusion in a calix[5]arene. The structures of a 1,2,3-alternate p-tert-butylcalix[4]arene derivative, where two of the ethereal chains partly fill the molecular cavity (Ungaro, Pochini, Andreetti \& Domiano, 1982), and of the partial-cone conformer of dicyanomethoxydimethoxycalix [4]arene, where one of the nitrile groups partly fills the calixarene cavity (Guelzim et al., 1993), both as self-inclusion monomers, have been reported previously.

Molecular dimensions (summarized in Table 2) are unexceptional and serve to establish the structure.

\section{Experimental \\ Crystal data \\ $\mathrm{C}_{55} \mathrm{H}_{70} \mathrm{O}_{5} .0 .3 \mathrm{C}_{6} \mathrm{H}_{14}$ \\ $M_{r}=837.0$ \\ Monoclinic \\ $\mathrm{C} 2 / \mathrm{c}$ \\ $a=34.687$ (3) ^ \\ $b=13.396(2) \AA$ \\ $c=25.721$ (2) $\AA$ \\ $\beta=113.518(7)^{\circ}$ \\ $V=10959$ (2) $\AA^{3}$ \\ $Z=8$}

$D_{x}=1.014 \mathrm{Mg} \mathrm{m}^{-3}$

Mo $K \alpha$ radiation

$\lambda=0.71073 \AA$

Cell parameters from 25 reflections

$\theta=12-15^{\circ}$

$\mu=0.06 \mathrm{~mm}^{-1}$

$T=293 \mathrm{~K}$

Block

$0.55 \times 0.25 \times 0.25 \mathrm{~mm}$

Colourless

\section{Data collection}

Nonius CAD-4 diffractometer $\omega 2 \theta$ scans

Absorption correction: none
6439 measured reflections 6274 independent reflections 2591 observed reflections

$\left[I_{\text {net }}>3.0 \sigma\left(I_{\text {net }}\right)\right]$

\section{Refinement}

Refinement on $F$

$R=0.066$

$w R=0.092$

$S=1.48$

2591 reflections

564 parameters

$\mathrm{H}$ atoms refined as riding,

$\mathrm{C}-\mathrm{H}$ and $\mathrm{O}-\mathrm{H} 0.95 \AA$ $w=1 /\left[\sigma^{2}(F)+0.0025 F^{2}\right]$ $(\Delta / \sigma)_{\max }=0.04$
3 standard reflections frequency: $60 \mathrm{~min}$ intensity variation: none

Table 1. Fractional atomic coordinates and equivalent isotropic displacement parameters $\left(\AA^{2}\right)$

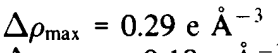

$\Delta \rho_{\min }=-0.18 \mathrm{e}^{-3}$

Extinction correction: Larson (1970)

Extinction coefficient: 23325 (7468)

Atomic scattering factors from International Tables for X-ray Crystallography (1974, Vol. IV, Table 2.2B)

\begin{tabular}{|c|c|c|c|c|}
\hline & & $\frac{1}{3} \sum_{i} \sum_{j} U_{i j} a_{i}^{*}$ & $a_{j}$. & \\
\hline & $x$ & $y$ & $z$ & $U_{\mathrm{eq}}$ \\
\hline $\mathrm{O} 1 A$ & $0.3422(2)$ & $0.1547(4)$ & $0.8986(3)$ & $0.064(4)$ \\
\hline $\mathrm{Cl} A$ & $0.3097(3)$ & $0.1171(6)$ & $0.8511(4)$ & $0.049(6)$ \\
\hline $\mathrm{C} 2 \mathrm{~A}$ & $0.3181(3)$ & $0.0871(6)$ & $0.8048(4)$ & $0.053(7)$ \\
\hline $\mathrm{C} 3 A$ & $0.2849(3)$ & $0.0469(7)$ & $0.7590(4)$ & $0.064(8)$ \\
\hline $\mathrm{C} 4 \mathrm{~A}$ & 0.2451 (3) & $0.0325(6)$ & $0.7580(4)$ & $0.058(7)$ \\
\hline $\mathrm{C} 5 \mathrm{~A}$ & $0.2400(3)$ & $0.0621(6)$ & $0.8064(4)$ & $0.059(7)$ \\
\hline $\mathrm{C} 6 A$ & $0.2715(3)$ & $0.1033(6)$ & $0.8538(4)$ & $0.048(6)$ \\
\hline$C 7 A$ & $0.3619(3)$ & $0.0868(7)$ & $0.8063(4)$ & $0.066(7)$ \\
\hline $\mathrm{C} 8 A$ & $0.2103(4)$ & $-0.0179(8)$ & $0.7089(4)$ & $0.077(8)$ \\
\hline C9A & $0.2184(4)$ & $-0.0279(12)$ & $0.6566(5)$ & $0.149(13)$ \\
\hline $\mathrm{C} 10 \mathrm{~A}$ & $0.1708(5)$ & $0.0458(17)$ & $0.6913(7)$ & $0.201(18)$ \\
\hline $\mathrm{C} 11 A$ & $0.1988(7)$ & $-0.1108(15)$ & $0.7259(6)$ & $0.247(21)$ \\
\hline $\mathrm{O} 1 B$ & $0.3211(2)$ & $0.2726(4)$ & $0.9727(2)$ & $0.055(4)$ \\
\hline $\mathrm{ClB}$ & $0.2788(3)$ & $0.2947(6)$ & $0.9496(3)$ & $0.040(6)$ \\
\hline $\mathrm{C} 2 B$ & $0.2506(3)$ & $0.2247(6)$ & $0.9147(3)$ & $0.044(6)$ \\
\hline $\mathrm{C} 3 B$ & $0.2083(3)$ & $0.2506(7)$ & $0.8908(4)$ & $0.054(6)$ \\
\hline$C 4 B$ & $0.1928(3)$ & $0.3415(7)$ & $0.9001(4)$ & $0.051(7)$ \\
\hline $\mathrm{C} 5 \mathrm{~B}$ & $0.2224(3)$ & $0.4053(6)$ & $0.9381(4)$ & $0.055(7)$ \\
\hline$C 6 B$ & $0.2652(3)$ & $0.3832(6)$ & $0.9638(3)$ & $0.042(6)$ \\
\hline$C 7 B$ & $0.2640(3)$ & $0.1208(6)$ & $0.9069(3)$ & $0.050(6)$ \\
\hline$C 8 B$ & $0.1472(3)$ & $0.3688(8)$ & $0.8719(4)$ & $0.067(7)$ \\
\hline $\mathrm{C} 9 \mathrm{~B}$ & $0.1194(4)$ & $0.2801(13)$ & $0.8510(8)$ & $0.194(17)$ \\
\hline$C 10 B$ & $0.1411(4)$ & $0.4341(18)$ & $0.8252(8)$ & $0.293(24)$ \\
\hline$C 11 B$ & $0.1311(4)$ & $0.4103(14)$ & $0.9141(7)$ & $0.177(16)$ \\
\hline $\mathrm{O} I \mathrm{C}$ & $0.3793(2)$ & $0.4211(4)$ & $1.0176(2)$ & $0.057(4)$ \\
\hline $\mathrm{ClC}$ & $0.3605(3)$ & $0.5133(6)$ & 0.9994 & $0.046(6)$ \\
\hline $\mathrm{C} 2 \mathrm{C}$ & $0.3192(3)$ & $0.5292(6)$ & $0.9904(3)$ & $0.041(6)$ \\
\hline $\mathrm{C} 3 \mathrm{C}$ & $0.3015(3)$ & $0.6197(6)$ & $0.9682(3)$ & $0.049(6)$ \\
\hline $\mathrm{C} 4 \mathrm{C}$ & $0.3238(3)$ & $0.6954(6)$ & $0.9557(4)$ & $0.054(7)$ \\
\hline $\mathrm{C} 5 \mathrm{C}$ & $0.3653(3)$ & $0.6780(6)$ & $0.9695(4)$ & $0.054(7)$ \\
\hline $\mathrm{C} 6 \mathrm{C}$ & 0.3853 & $0.5900(7)$ & $0.9921(3)$ & $0.049(6)$ \\
\hline $\mathrm{C} 7 \mathrm{C}$ & 0.2945 (3) & $0.4526(6)$ & $1.0082(4)$ & $0.052(6)$ \\
\hline $\mathrm{C} 8 \mathrm{C}$ & $0.3023(4)$ & $0.7945(7)$ & $0.9291(5)$ & $0.085(10)$ \\
\hline $\mathrm{C} 9 \mathrm{Cl}$ & 0.33399 & 0.86121 & 0.91974 & $0.23(6)$ \\
\hline $\mathrm{C} 10 \mathrm{Cl}$ & 0.26674 & 0.77459 & 0.87265 & $0.13(3)$ \\
\hline $\mathrm{C} 11 \mathrm{Cl}$ & 0.28569 & 0.84541 & 0.96746 & $0.12(3)$ \\
\hline $\mathrm{C} 9 \mathrm{C2}$ & 0.25654 & 0.79127 & 0.91793 & $0.19(4)$ \\
\hline $\mathrm{C} 10 \mathrm{C} 2$ & 0.32304 & 0.87914 & 0.96833 & $0.29(6)$ \\
\hline $\mathrm{C} 11 \mathrm{C2}$ & 0.30683 & 0.81080 & 0.87359 & $0.17(4)$ \\
\hline $\mathrm{O} 1 D$ & $0.4347(2)$ & $0.3908(4)$ & $0.9603(2)$ & $0.065(4)$ \\
\hline $\mathrm{C} 1 D$ & $0.4470(2)$ & $0.4729(8)$ & $0.9387(4)$ & $0.053(6)$ \\
\hline $\mathrm{C} 2 D$ & $0.4455(2)$ & $0.5672(8)$ & $0.9616(4)$ & $0.055(7)$ \\
\hline $\mathrm{C} 3 D$ & $0.4569(3)$ & $0.6488(8)$ & $0.9379(5)$ & $0.076(8)$ \\
\hline$C 4 D$ & $0.4701(3)$ & $0.6401(10)$ & $0.8938(5)$ & $0.085(9)$ \\
\hline$C 5 D$ & $0.4735(3)$ & $0.5452(11)$ & $0.8750(5)$ & $0.080(9)$ \\
\hline$C 6 D$ & $0.4631(3)$ & $0.4609(8)$ & $0.8983(4)$ & $0.062(7)$ \\
\hline$C 7 D$ & $0.4319(3)$ & $0.5796(6)$ & $1.0098(4)$ & $0.059(6)$ \\
\hline$C 8 D$ & $0.4806(5)$ & $0.7347(11)$ & $0.8679(7)$ & $0.120(12)$ \\
\hline
\end{tabular}




$\begin{array}{lllll}\text { C9D } & 0.4473(6) & 0.8107(13) & 0.8530(10) & 0.219(22) \\ \text { C10D } & 0.4909(9) & 0.7155(14) & 0.8175(10) & 0.27(3) \\ \text { C11D } & 0.5181(7) & 0.7800(15) & 0.9102(10) & 0.235(22) \\ \text { O1E } & 0.41679(17) & 0.2136(5) & 0.89548(24) & 0.066(5) \\ \text { C1E } & 0.4103(3) & 0.2388(7) & 0.8404(4) & 0.054(7) \\ \text { C2E } & 0.4339(3) & 0.3131(7) & 0.8303(4) & 0.059(7) \\ \text { C3E } & 0.4251(3) & 0.3408(8) & 0.7751(5) & 0.069(8) \\ \text { C4E } & 0.3944(3) & 0.2951(8) & 0.7286(4) & 0.066(8) \\ \text { C5E } & 0.3731(3) & 0.2149(7) & 0.7401(4) & 0.064(7) \\ \text { C6E } & 0.3810(3) & 0.1839(7) & 0.7961(4) & 0.058(7) \\ \text { C7E } & 0.4706(3) & 0.3599(9) & 0.8790(4) & 0.074(8) \\ \text { C8E } & 0.3859(4) & 0.3324(10) & 0.6699(5) & 0.089(10) \\ \text { C9E } & 0.4246(4) & 0.3502(16) & 0.6611(5) & 0.188(17) \\ \text { C10E } & 0.3609(6) & 0.4275(14) & 0.6582(6) & 0.189(18) \\ \text { C11E } & 0.3607(6) & 0.2594(14) & 0.6244(6) & 0.194(18) \\ \text { C1 } H & 0.0052(13) & 0.431(3) & 0.218(2) & 0.21(2) \dagger \\ \text { C2H } & -0.0073(15) & 0.412(4) & 0.153(3) & 0.20(2) \dagger \\ \text { C3H } & 0.0 & 0.42083 & 1 & 0.20(6) \dagger \\ \text { C4H } & -0.01923 & 0.44851 & 0.17917 & 0.18(3) \dagger \\ \text { C5H } & -0.01595 & 0.41524 & 0.10916 & 0.16(3) \dagger \\ \text { C6H } & -0.03277 & 0.41739 & 0.06527 & 0.21(3) \dagger \\ \text { C7H } & -0.03958 & 0.42880 & 0.02083 & 0.22(3) \dagger\end{array}$

† These seven sites about a twofold axis are associated with a very disordered $n$-hexane molecule which was assigned an overall occupancy of 0.3 on the basis of peak heights in Fourier and difference Fourier maps.

Table 2. Selected bond lengths $(\AA)$ (range and mean) for molecule $(\mathrm{I})$

\begin{tabular}{lll} 
& \multicolumn{1}{c}{ Range } & Mean \\
$\mathrm{C}_{\mathrm{ar}}-\mathrm{O}_{\text {phenolic }}$ & $1.373(11)-1.387(10)$ & $1.382(11)$ \\
$\mathrm{C}_{\mathrm{ar}}-\mathrm{C}_{\mathrm{arr}}$ & $1.358(13)-1.417(14)$ & $1.386(14)$ \\
$\mathrm{C}_{\mathrm{ar}}-\mathrm{C}_{s p}{ }^{3}$ & $1.497(12)-1.543(13)$ & $1.519(13)$ \\
$\mathrm{C}_{s p^{3}}-\mathrm{C}_{s p}{ }^{3}$ & $1.429(20)-1.520(24)$ & $1.480(21)^{\star}$
\end{tabular}

* The disordered methyl $\mathrm{C}$ atoms of the tert-butyl group on ring $C$ were not included in the calculation.

Table 3. Summary of the O...O separations $(\AA)$ (range and mean) and aromatic ring orientations $\left(^{\circ}\right)$ (range and mean) for the reported calix[5]arenes

\begin{tabular}{cccccc}
\multicolumn{2}{c}{$\mathrm{O} \cdots \mathrm{O}$} & \multicolumn{4}{c}{ Aromatic ring orientations } \\
Range & Mean & Range & Mean & Molecule \\
$2.735(7)-2.880(8)$ & $2.793(8)$ & $118.6(3)-149.7(3)$ & $133.6(3)$ & (I) \\
$2.828(9)-2.856(10)$ & $2.841(10)$ & $126.5(8)-139.4(6)$ & $131.8(8)$ & (II) \\
$2.750(11)-2.881(11)$ & $2.801(10)$ & $122.7(2)-145.4(2)$ & $130.7(2)$ & (IV) \\
$2.696(8)-2.822(6)$ & $2.761(10)$ & $130.3(1)-145.4(1)$ & $135.4(1)$ & (V)
\end{tabular}

References: (I) present work; (II) Conuzzi et al. (1982); (IV) Perrin \& Lecocq (1991); (V) Perrin \& Lecocq (1991).

Table 4. The $\varphi$ and $\chi$ torsion angle values $\left({ }^{\circ}\right)$, which define the conformation of (I) according to the scheme proposed by Ugozzoli \& Andreetti (1992)

$\begin{array}{lcc} & \varphi & \chi \\ A-B & 86.3(7) & -96.5(7) \\ B-C & 84.7(7) & -97.2(7) \\ C-D & 93.1(8) & -75.6(7) \\ D-E & 80.7(8) & -93.0(9) \\ E-A & 105.7(9) & -85.4(8)\end{array}$

The pentahydroxy-p-tert-butylcalix [5]arene was synthesized by a procedure described by Stewart \& Gutsche (1993). Suitable crystals were obtained on recystallization from $\mathrm{CHCl}_{3}$ $n$-hexane. Molecule (I) crystallized in the monoclinic system; the systematic absences $(h k l, h+k=2 n+1 ; h 0 l, l=2 n+$
1) allow the space group to be either $C 2 / c$ or $C c$; the former was assumed and confirmed by the analysis. It was clear from early difference maps that the methyl $C$ atoms of the tertbutyl group on aromatic ring $C$ were disordered equally over two sites (a relatively common occurrence); this was allowed for by using six half-occupancy $\mathrm{C}$ atoms, positioned geometrically $[\mathrm{C}-\mathrm{C} 1.50$ (1) $\AA$ ] , and refining only their thermal parameters. Difference density maps in the plane of the five $O$ atoms showed clearly that the hydroxyl $\mathrm{H}$ atoms were disordered over two orientations; this was allowed for by positioning each half-occupancy $\mathrm{H}$ atom along the $\mathrm{O} \cdots \mathrm{O}$ vector $[\mathrm{O}-\mathrm{H} 0.95 \AA]$. The $\mathrm{H}$ atoms attached to the $\mathrm{C}$ atoms were positioned geometrically $[\mathrm{C}-\mathrm{H} 0.95 \AA]$ and all were included as riding atoms in the structure-factor calculations. It was evident at an intermediate stage of the refinement that there was a disordered partialoccupancy $n$-hexane solvent of crystallization in the lattice, situated about a twofold axis. The two major peaks were refined and the minor peaks were included at the positions in which they were located from the difference maps and only their thermal parameters were allowed to refine isotropically. $\mathrm{H}$ atoms were not included for this disordered solvent molecule. Data collection: CAD-4 Software (Enraf-Nonius, 1989). Cell refinement: CAD-4 Software. Data reduction: NRCVAX DATRD2 (Gabe, Le Page, Charland, Lee \& White, 1989). Program(s) used to solve structure: NRCVAX SOLVER. Program(s) used to refine structure: NRCVAX LSTSQ. Molecular graphics: NRCVAX ORTEPII (Johnson, 1976); PLUTON (Spek, 1991). Software used to prepare material for publication: NRCVAX TABLES.

GF thanks NSERC Canada for research grants. The authors thank Professor C. D. Gutsche for a preliminary communication of the synthetic procedure for the tertbutylcalix[5]arene.

Lists of structure factors, anisotropic displacement parameters, H-atom coordinates and complete geometry have been deposited with the British Library Document Supply Centre as Supplementary Publication No. SUP $71470(28 \mathrm{pp}$.). Copies may be obtained through The Technical Editor, International Union of Crystallography, 5 Abbey Square, Chester CH1 2HU, England. [CIF reference: MU1052]

\section{References}

Allen, F. H., Kennard, O. \& Taylor, R. (1983). Acc. Chem. Res. 16, 146153.

Böhmer, V., Ferguson, G., Gallagher, J. F., Lough, A. J., McKervey, M. A., Madigan, E., Moran, M., Phillips J. \& Williams, G. J. B. (1993). J. Chem. Soc. Perkin Trans. 1. pp. 1521-1527.

Coruzzi, M., Andreetti, G. D., Bocchi, V., Pochini, A. \& Ungaro, R. (1982). J. Chem. Soc. Perkin Trans. 2, pp. 1133-1138.

Enraf-Nonius (1989). CAD-4 Software. Version 5.0. Enraf-Nonius, Delft, The Netherlands.

Ferguson, G., Gallagher, J. F., McKervey, M. A. \& Moran, M. B. (1993). In preparation.

Ferguson, G., Gallagher, J. F. \& Pappalardo, S. (1993). J. Incl. Phenom. Mol. Recognit. Chem. 14, 349-356.

Gabe, E. J., Le Page, Y., Charland, J.-P., Lee, F. L. \& White, P. S. (1989). J. Appl. Cryst. 22, 384-387.

Guelzim, A., Khrifi, S., Baert, F., Loeber, C., Asfari, Z., Matt, D. \& Vicens, J. (1993). Acta Cryst. C49, 72-75.

Gutsche, C. D. (1989). In Calixarenes. Cambridge: Royal Society of Chemistry. 
Johnson, C. K. (1976). ORTEPII. Report ORNL-5138. Oak Ridge National Laboratory, Tennessee, USA.

Juneja, R. K., Robinson, K. D., Orr, G. W., Dubois, R. H., Belmore, K. A., Atwood, J. L., Ripmeester, J. M. \& Ratcliffe, J. (1992). J. Incl. Phenom. Mol. Recognit. Chem. 13, 93-96.

Kraft, D., Böhmer, V., Vögt, W., Ferguson G. \& Gallagher, J. F. (1993). In preparation.

Larson, A. C. (1970). Crystallographic Computing, edited by F. R. Ahmed, S. R. Hall \& C. P. Huber, pp. 291-294. Copenhagen: Munksgaard.

McKervey, M. A., Seward, E., Ferguson, G. \& Ruhl, B. L. (1986). J. Org. Chem. 51, 3581-3584.

Pappalardo, S., Giunta, L., Foti, L., Ferguson, G., Gallagher, J. F. \& Kaitner, B. (1992). J. Org. Chem. 57, 2611-2624.

Perrin, M. \& Lecocq, S. (1991). J. Incl. Phenom. Mol. Recognit. Chem. 11, 171-183.

Spek, A. L. (1991). PLUTON. Molecular Graphics Program. Univ. of Utrecht, The Netherlands.

Stewart, D. R. \& Gutsche, C. D. (1993). Org. Prep. Proced. Int. In the press.

Ugozzoli, F. \& Andreetti, G. D. (1992). J. Incl. Phenom. Mol. Recognit. Chem. 13, 337-348.

Ungaro, R., Pochini, A., Andreetti, G. D. \& Domiano, P. (1982). J. Incl. Phenom. Mol. Recognit. Chem. 3, 35-42.

Vicens, J. \& Böhmer, V. (1991). In Calixarenes: a Versatile Class of Macrocyclic Compounds. Dordrecht: Kluwer Academic Press.

Weber, E. \& Josel, H. P. (1983). J. Incl. Phenom. Mol. Recognit. Chem. 1, 79-85.

Acta Cryst. (1994). C50, 77-78

\section{Structure of 2,2,6,6-Tetramethyl-4- oxaheptanedinitrile}

\section{George Ferguson, John F. Gallagher, $\dagger$ \\ AlAN J. MCAlEES \\ Department of Chemistry and Biochemistry, \\ University of Guelph, Guelph, Ontario, \\ Canada NIG 2WI}

(Received 4 May 1993; accepted 6 July 1993)

\begin{abstract}
The title molecule, $\mathrm{C}_{10} \mathrm{H}_{16} \mathrm{~N}_{2} \mathrm{O}(\mathrm{I})$, has approximate mirror symmetry with the main chain fully extended. The main torsion angles defining the molecular conformation are $\mathrm{O}-\mathrm{C}_{s p^{3}}-\mathrm{C}_{s p^{3}}-\mathrm{C}_{s p} 58.9(4)$ and $-60.5(4)^{\circ}$. The mean principal dimensions include $\mathrm{N} \equiv \mathrm{C}_{s p} 1.13(1)$, $\mathrm{C}_{s p}-\mathrm{C}_{s p^{3}} 1.46(1)$ and $\mathrm{C}_{s p^{3}}-\mathrm{O} 1.410(6) \AA$. The two nitrile groups are oriented cis to one another $\left[\mathrm{N} \equiv \mathrm{C}_{s p} \cdots\right.$ $\left.\mathrm{C}_{s p} \equiv \mathrm{N} 4.4(5)^{\circ}\right]$ and the intramolecular $\mathrm{N} \cdots \mathrm{N}$ separation is 4.70 (1) $\AA$.
\end{abstract}

† Present address: University Chemical Laboratories, Lensfield Road, Cambridge CB2 1EW, England.

\section{Comment}

The structural determination of 2,2,6,6-tetramethyl-4oxaheptanedinitrile (I) was undertaken as part of a research program directed towards the development of new macrocyclic ligands for use in the synthesis of transition-metal complexes (Ferguson, McAlees, McCrindle, Restivo \& Roberts, 1977; McCrindle, Ferguson, McAlees, Parvez, Ruhl, Stephenson \& Wieckowski, 1986; McCrindle, Ferguson, McAlees, Parvez \& Stephenson, 1982, 1983; McCrindle, Ferguson, McAlees \& Roberts, 1981; McCrindle, Ferguson \& Parvez, 1984; McAlees \& McCrindle, 1981).

The crystal structure determination establishes the overall conformation and the orientation of the two nitrile moieties in the solid state, Fig. 1. (I) has pseudo-mirror symmetry with the mirror plane passing through the central ether $\mathrm{O}$ atom; the two nitrile groups are oriented $c i s$ and are almost parallel to one another $\left[\mathrm{N} \equiv \mathrm{C}_{s p} \cdots \mathrm{C}_{s p} \equiv \mathrm{N}\right.$ $\left.4.4(5)^{\circ}\right]$, and to the $a$ axis. The angle between the $\mathrm{C} 32-$ and $\mathrm{C} 72-\mathrm{C}_{s p^{3}}-\mathrm{C}_{s p} \equiv \mathrm{N}$ planes is $2.6(6)^{\circ}$. The principal dimensions include: $\mathrm{N} \equiv \mathrm{C}_{s p} 1.127$ (10) and 1.129 (10) $\AA$; $\mathrm{C}_{s p}-\mathrm{C}_{s p^{3}} 1.464(10)$ and $1.467(10) \AA ; \mathrm{C}_{s p^{3}}-\mathrm{C}_{s p^{3}}$ in the range $1.506(8)-1.532(8) \AA$ with a mean value of 1.512 (7) $\AA ; \mathrm{C}_{s p^{3}}-\mathrm{O} 1.409$ (6) and 1.412 (6) $\AA$. The main torsion angles defining the molecular conformation are $\mathrm{O}-\mathrm{C}_{s p^{3}}-\mathrm{C}_{s p^{3}}-\mathrm{C}_{s p} 58.9(4)$ and $-60.5(4)^{\circ}$. The distance between $\mathrm{N} 1$ and N9 is 4.70 (1) $\AA$, and this makes the molecule a potentially useful bridging ligand in metalcluster chemistry. Full details of other molecular dimensions are available as supplementary material.

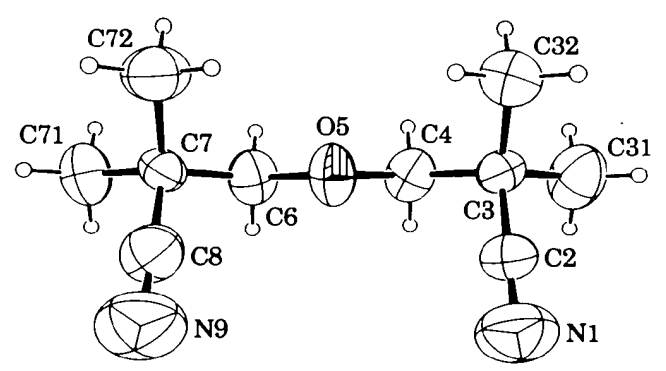

Fig. 1. An ORTEPII view of molecule (I), with the non-H atoms shown with their thermal ellipsoids drawn at the $50 \%$ probability level. For clarity, the $\mathrm{H}$ atoms are drawn as spheres of arbitrary size.

\section{Experimental \\ Crystal data}

$\mathrm{C}_{10} \mathrm{H}_{16} \mathrm{~N}_{2} \mathrm{O}$

$M_{r}=180.25$

Monoclinic

$P 2_{1} / c$

$a=6.6495$ (4) ^

$b=9.8627(7) \AA$

$c=17.6172(12) \AA$

$\beta=91.286(5)^{\circ}$

$$
\begin{aligned}
& D_{x}=1.04 \mathrm{Mg} \mathrm{m}^{-3} \\
& \text { Mo } K \alpha \text { radiation } \\
& \lambda=0.71073 \AA \\
& \text { Cell parameters from } 25 \\
& \quad \text { reflections } \\
& \theta=7.5-15.6^{\circ} \\
& \mu=0.064 \mathrm{~mm}^{-1} \\
& T=293 \mathrm{~K} \\
& \text { Block }
\end{aligned}
$$

